\title{
Limitation of Mitragynine Biosynthesis in Mitragyna speciosa (Roxb.) Korth. through Tryptamine Availability
}

\author{
Tossaton Charoonratana ${ }^{\text {a }}$, Juraithip Wungsintaweekul ${ }^{\mathrm{a}, *}$, Pathamaporn \\ Pathompak $^{\mathrm{a}}$, Milen I. Georgiev ${ }^{\mathrm{b}}$, Young Hae Choi ${ }^{\mathrm{b}}$, and Robert Verpoorte \\ a Department of Pharmacognosy and Pharmaceutical Botany, Faculty of Pharmaceutical \\ Sciences, Prince of Songkla University, Hat Yai, Songkhla 90112, Thailand. \\ Fax:+6674428220. E-mail: juraithip.w@psu.ac.th \\ b Natural Products Laboratory, Institute of Biology, Leiden University, Leiden, \\ 55 Einsteinweg, 2300 RA Leiden, The Netherlands \\ * Author for correspondence and reprint requests \\ Z. Naturforsch. 68c, 394-405 (2013); received November 30, 2012/August 8, 2013
}

Metabolite profiles of Mitragyna speciosa were determined by means of ${ }^{1} \mathrm{H}$ NMR-based and HPLC-based analyses. The results indicated that high contents of secologanin, caffeic acid, gallic acid, epigallocatechin, and mitragynine were accumulated in leaves. In $M$. speciosa, feedings of tryptamine, tryptophan, phenylalanine or tyrosine significantly increased the mitragynine contents. Feedings of tryptamine and loganin also enhanced the mitragynine accumulation, but feeding of loganin only did not affect the mitragynine level. The mRNA levels of anthranilate synthase alpha subunit $(A S A)$, tryptophan decarboxylase $(T D C)$, and strictosidine synthase $(S T R)$ were measured by quantitative real-time polymerase chain reaction (RT-qPCR) in control plants and those exposed to methyl jasmonate (MJ; $10 \mu \mathrm{M})$. All genes responded to MJ after a 24-h treatment. The mitragynine contents were also enhanced and corresponded to the transcript levels. From the present results we conclude that a high content of secologanin together with a undetectable level of tryptamine in $M$. speciosa feature the limitation of mitragynine biosynthesis. Additionally, expression of all the genes limits production of an essential precursor for mitragynine production.

Key words: Mitragyna speciosa, Mitragynine, Tryptamine

\section{Introduction}

Mitragyna speciosa (Roxb.) Korth. (Rubiaceae) is a tropical tree distributed in Southeast Asia, mainly in Thailand and Malaysia. The plant is generally known as 'kratom' in Thailand and 'ketum' in Malaysia (Singh, 1932). M. speciosa produces various kinds of monoterpenoid indole alkaloids (MIAs). Valuable MIAs are mitragynine and 7-hydroxymitragynine. Mitragynine, which accumulates in M. speciosa leaves, has been traditionally used as an opium substitute, and for treating diarrhea and cough in Thailand (Suwanlert, 1975; Thongpraditchote et al., 1998). Owing to its antinociceptive activity as well as its low addictive properties, mitragynine has attracted much scientific interest. In addition, 7-hydroxymitragynine was found to be 10 -fold more potent than morphine, the classical opiate (Kikura-Hanajiri et al., 2009). As with other metabolites found in plants, mitragynine is produced only in low yields of about $0.81 \%(\mathrm{w} / \mathrm{w})$ of dried leaves (Ponglux et al., 1994). Therefore, the principle aim of the experiments described here was to study the regulation of the mitragynine biosynthetic pathway. This knowledge might be further used to develop strategies for the metabolic engineering of $M$. speciosa to obtain a more efficient production of mitragynine or 7-hydroxymitragynine. Both metabolites and transcription profiles have been used as tools to obtain insight into the mitragynine biosynthesis. Metabolite profiling may provide the most useful information about the state and dynamics of the metabolic pathways of an organism at a given time point. Moreover, a metabolite profile represents the endpoint of gene expression at a particular time, and changes in the metabolite concentrations are expected to correlate with changes in the transcript levels (Sumner et al., 2011).

The biosynthesis of MIAs involves several enzymatic steps that are distributed among several subcellular compartments. In M. speciosa, mitragynine and 7-hydroxymitragynine originate from strictosidine, which is the condensation product 
of secologanin from the terpenoid pathway and tryptamine from the shikimate pathway (Rueffer et al., 1978; Nagakura et al., 1979). Recently, the regulation of $M$. speciosa strictosidine synthase has been investigated and found to respond to high concentrations of salicylic acid (Jumali et al., 2011). Application of methyl jasmonate and yeast extract to $M$. speciosa shoot cultures increased the transcript levels of tryptophan decarboxylase and strictosidine synthase (Wungsintaweekul et al., 2012). Since mitragynine is a compound of medicinal importance, the regulation of the mitragynine biosynthesis is an attractive area of research for advances in biotechnological applications. Thus, in the present study, we report the transcript profiles of the genes involved in the early steps of mitragynine biosynthesis. The limitation of mitragynine production was assessed by establishing metabolite profiles and by precursor feeding.

\section{Experimental}

\section{Chemicals}

Loganin, secologanin, tryptamine, and tryptophan were from Sigma-Aldrich (Singapore). Lloyd \& McCown woody plant medium (WPM; L449) was from PhytoTechnology Laboratories (Lenexa, KS, USA). $N$-Benzyladenine (BA) was from Fluka Chemie (Buchs, Switzerland). Thidiazuron (TDZ) was from Supelco (Bellefonte, PA, USA). Authentic mitragynine was isolated from the leaves of $M$. speciosa as described previously (Janchawee et al., 2007). Its structure was confirmed by ${ }^{13} \mathrm{C}$ NMR spectrometry. The purity was approximately $98 \%$, as determined by high-performance liquid chromatography (HPLC).

\section{Plant materials}

M. speciosa seeds were collected from Hat Yai district, Songkhla, Thailand. The seeds were surface-sterilized by rinsing with $70 \%(\mathrm{v} / \mathrm{v})$ ethanol for 5 min, rinsing with $20 \%(\mathrm{v} / \mathrm{v})$ Clorox $^{\circledR}$ for $5 \mathrm{~min}$, and finally rinsing with sterile distilled water. Sterilized seeds were germinated on hormonefree WPM medium and incubated at $25^{\circ} \mathrm{C}$ under long-day conditions ( $16 \mathrm{~h} \mathrm{light} / 8 \mathrm{~h}$ dark). After 2 months, the plantlets were ready for analysis of metabolites, feeding precursors, and treatment with methyl jasmonate (MJ).

The shoot cultures of $M$. speciosa were initiated from axillary buds of the two-month-old $M$. speciosa plantlets as described previously (Wungsintaweekul et al., 2012). The axillary buds were cut off and placed on WPM medium supplemented with $2 \mathrm{mg} / \mathrm{L} \mathrm{TDZ}$ and $1 \mathrm{mg} / \mathrm{L}$ BA. The shoot culture was maintained at $25^{\circ} \mathrm{C}$ under light for $16 \mathrm{~h} / \mathrm{d}$. These shoot cultures were used as starting materials for feeding precursors.

\section{NMR-based metabolite analysis}

Leaves of M. speciosa were harvested, immediately ground in liquid nitrogen, lyophilized, and divided into $50-\mathrm{mg}$ lots to be ready for ${ }^{1} \mathrm{H}$ NMR analysis. A sample was transferred to a $2-\mathrm{mL}$ microtube, to which $0.75 \mathrm{~mL} \mathrm{CD} \mathrm{CD}_{3} \mathrm{OD}$ and $0.75 \mathrm{~mL}$ $\mathrm{D}_{2} \mathrm{O}\left(\mathrm{KH}_{2} \mathrm{PO}_{4}\right.$ buffer, $\mathrm{pH}$ 6.0) were added. The mixture was vortexed at room temperature for $1 \mathrm{~min}$, ultrasonicated for $20 \mathrm{~min}$, and then centrifuged at $15,500 \times g$ at room temperature for $20 \mathrm{~min}$. The supernatant was then transferred to a 5-mm NMR tube and used for the NMR analyses. ${ }^{1} \mathrm{H}$ NMR and 2D $J$-resolved spectra were recorded at $25^{\circ} \mathrm{C}$ on a $500-\mathrm{MHz}$ Bruker DMX-500 spectrometer (Bruker, Karlsruhe, Germany). $\mathrm{CD}_{3} \mathrm{OD}$ was used as the internal lock. The resulting spectra were manually phased and baseline-corrected, and referenced to the internal standard $[0.01 \%$ (w/w) trimethylsilanepropionic acid (TMSP)] at $0.0 \mathrm{ppm}$, using Topspin (version 2.1; Bruker). The 2D $J$-resolved NMR spectra were acquired using 8 scans per 128 increments of $F_{1}$ and $8 \mathrm{k}$ increments for $F_{2}$ using spectral widths of $500 \mathrm{~Hz}$ in $F_{2}$ (chemical shift axis) and $66 \mathrm{~Hz}$ in $F_{1}$ (spin-spin coupling constant axis). The $J$-resolved spectra were tilted by $45^{\circ}$, symmetrized about $F_{1}$, and then calibrated using XWIN NMR software. The obtained NMR spectra were compared to the NMR spectra database of the Natural Products Laboratory, Institute of Biology, Leiden University, Leiden, The Netherlands. Chemical shifts $(\delta)$ are reported in ppm and coupling constants $(J)$ are given in $\mathrm{Hz}$.

\section{HPLC-based metabolite analysis}

Freeze-dried $M$. speciosa leaves, roots, and stems $(50 \mathrm{mg})$ were separately extracted by reflux boiling with methanol at $70^{\circ} \mathrm{C}$ for $1 \mathrm{~h}$. The extracts were filtered, then washed twice with petroleum ether, and concentrated under reduced pressure. Samples were analysed immediately after extraction in order to avoid possible chemical degradation. 
HPLC analysis was carried out using an Agilent 1100 series system equipped with an Agilent 1100 series photodiode-array detector (San Francisco, CA, USA) and a fluorescence detector; data analysis was performed using Agilent software. Chromatographic separation was performed on a reverse phase VertiSep ${ }^{\mathrm{TM}}$ UPS C18 column $(250 \mathrm{~mm} \times 4.6 \mathrm{~mm}$ i.d., $5 \mu \mathrm{m}$ particle size $)$ (Vertical, Bangkok, Thailand) with a binary gradient mobile phase profile $[1.5 \mathrm{~mL} / \mathrm{min}$; acetonitrile/100 mм $\mathrm{H}_{3} \mathrm{PO}_{4}$ in water, $\mathrm{pH} 2.4$ (10:90 to 90:10, v/v, within $15 \mathrm{~min})]$. The identification of metabolites, including secologanin, tryptophan, tryptamine, and mitragynine, was based on the retention times and comparison of the absorption spectra with those of authentic standards. The wavelength for quantitative determination was set at $225 \mathrm{~nm}$ and $238 \mathrm{~nm}$ for mitragynine and secologanin, respectively. Tryptophan and tryptamine were quantified by fluorescence measurements with the excitation wavelength at $270 \mathrm{~nm}$ and the emission wavelength at $370 \mathrm{~nm}$. Quantification was repeated three times for each culture. The parameters of linearity, reproducibility, accuracy, and specificity of the method were evaluated.

\section{Precursor feeding}

Solutions of $1 \mathrm{mg} / \mathrm{mL}$ of tryptamine, tryptophan, tyrosine, and phenylalanine were separately prepared in $50 \%(\mathrm{v} / \mathrm{v})$ aqueous methanol, filtersterilized, and individually added to liquid WPM to $100 \mu \mathrm{g} / \mathrm{mL}$ final concentration. The 2-monthold plantlets were grown in the media under longday conditions for 1 month, the control samples were cultivated in WPM supplemented only with the corresponding amount of $50 \%(\mathrm{v} / \mathrm{v})$ aqueous methanol. After feeding for 1 month, the newly formed shoots of plantlets were collected in order to determine the amounts of metabolites by HPLC as mentioned above. Three samples of each experiment were separately prepared for analysis.

A feeding study of tryptamine, loganin, and a combination of both compounds on mitragynine production was performed in $M$. speciosa shoot cultures. A cluster of shoots (4-5 shoots/explant) was inoculated into $30 \mathrm{~mL}$ of liquid WPM supplemented with the same plant growth regulators. Stock solutions of tryptamine were prepared in ethanol at concentrations of $250 \mathrm{~mm}$ and $500 \mathrm{~mm}$. Stock solutions $(24 \mu \mathrm{L}$ each) were added to the shoot cultures to obtain final concentrations of $0.2 \mathrm{~mm}$ and $0.4 \mathrm{~mm}$, respectively. A stock solution of loganin was prepared in sterilized water (100 mM). Volumes of $60 \mu \mathrm{L}$ and $120 \mu \mathrm{L}$ of the solutions were added to achieve final concentrations of $0.2 \mathrm{~mm}$ and $0.4 \mathrm{~mm}$. Applications of tryptamine, loganin, or combinations of both were performed on the day of inoculation. The shoot cultures were incubated under culture conditions and shaking at $60 \mathrm{rpm}$. After 14 and $21 \mathrm{~d}$ of feeding, the shoot cultures were harvested, thoroughly washed with water, and the excess water absorbed by layers of tissue paper. The shoots were lyophilized and ground to powder. Samples were prepared for quantification of their mitragynine content.

\section{Methyl jasmonate (MJ) treatment}

Two-month-old plantlets were removed to liquid WPM. A stock solution of $1 \mathrm{~mm}$ MJ was prepared in $50 \%(\mathrm{v} / \mathrm{v})$ aqueous methanol. The MJ solution was added to obtain a final concentration of $10 \mu \mathrm{M}$ MJ. Plantlets were incubated for $12 \mathrm{~h}$ and $24 \mathrm{~h}$. Leaves and stems were excised. Samples were lyophilized and prepared for HPLC analysis. For quantitative real-time polymerase chain reaction (RT-qPCR) analysis, samples were immediately frozen and kept at $-80{ }^{\circ} \mathrm{C}$ until used.

\section{Transcription profile analysis by RT-qPCR}

The plant tissues were ground in liquid nitrogen. Total RNA was extracted using an RNeasy Plant Mini Kit (Qiagen, Hilden, Germany) by following the manufacturer's protocols, including the DNA elimination step. The RNA pattern was evaluated for intactness by electrophoresis in $1.2 \%(\mathrm{w} / \mathrm{v})$ agarose gel. RNA amounts were determined by the absorbance at $260 \mathrm{~nm}\left(A_{260}\right)$. The $A_{260} / A_{280}$ ratios of the purified RNA samples were in the range of 1.8 to 2.1. The first-strand DNA was then synthesized by reverse transcriptase. The reverse transcription was carried out on $1.0 \mu \mathrm{g}$ of total RNA using SuperScript III reverse transcriptase (Invitrogen, Carlsbad, CA, USA) following the user's manual.

The RT-qPCR was used to determine the transcription of $M$. speciosa anthranilate synthase alpha subunit isoform 1 and isoform 2 ( $A S A 1$ and ASA2; GenBank ID: JQ775867 and JQ775866), tryptophan decarboxylase (TDC; GenBank ID: JN643922.1), and strictosidine synthase (STR; GenBank ID: EU288197.1) genes from the non- 
Table I. List of primers used in RT-qPCR.

\begin{tabular}{|c|c|c|c|}
\hline Primer name & Primer efficiency $^{\mathrm{a}}$ & Nucleotide sequence $\left(5^{\prime} \rightarrow 3^{\prime}\right)$ & Amplicon size [bp] \\
\hline $18 \mathrm{~S}$ rRNAf & 0.88 & CAAAGCAAGCCTACGCTCTG & 530 \\
\hline 18S rRNAr & & CGCTCCACCAACTAAGAACG & \\
\hline EF1af & 0.97 & CTGGTGGAACAAGATGGATG & 270 \\
\hline EF1a1r & & CCAATACCACCAATCTTGTA & \\
\hline TUAf & 0.84 & ACGCTGTTGGTGGTGGTAC & 106 \\
\hline TUAr & & GAGAGGGGTAAACAGTGAATC & \\
\hline UBQ10f & 0.89 & ATCACCCTTGAAGTGGA & 194 \\
\hline UBQ10r & & GAAACCACCACGAAGAC & \\
\hline UBQ5f & 0.80 & GACGCTTCATCTCGTCC & 186 \\
\hline UBQ5r & & CCACAGGTTGCGTTAG & \\
\hline TEF2f & 0.81 & GGTGTGACGATGAAGAGTGATG & 129 \\
\hline TEF2r & & TGAAGGAGAGGGGAAGGTGAAAG & \\
\hline ASA1f & 0.97 & СТСТСТССАGTСТTСАТСТСС & 316 \\
\hline ASA1r & & САСААССАТСТССТТСААСТТСС & \\
\hline ASA2f & 0.97 & CCACAGTTTCTCCTCCGTCA & 311 \\
\hline ASA2r & & CTGTTCCACTCTTCGTCCTTG & \\
\hline TDC147S & 0.98 & GCTTTTTCGTCAAGACTTGCAAATTGGCTG & 361 \\
\hline TDC508A & & GTAACAAAGTAGCCACTTATGAGGGCTC & \\
\hline STR808S & 0.92 & GGAATTACTGTTACGCCTAGAGC & 245 \\
\hline STR1053A & & AGAAGAAGCCACTCCATTCAAAG & \\
\hline
\end{tabular}

a Primer efficiency was calculated from REST 2009 software V.2.0.13 (Qiagen).

induced and MJ-induced samples. The RT-qPCR was performed using specific primers (Table I) on an ABI PRISM ${ }^{\circledR} 7300$ Sequence Detector (Applied Biosystems, Foster City, CA, USA) with SYBR $^{\circledR}$ Green to monitor the synthesis of double-stranded DNA. The software program Vector $\mathrm{NTI}^{\circledR}$ (Invitrogen) was used to design and determine the propensity of primers to form dimers and to determine the optimal annealing temperature. The PCR reactions were carried out in a 96well plate, and the final PCR reaction volume was $20 \mu \mathrm{L}$, containing $1 \mathrm{x} \mathrm{SYBR}^{\circledR}$ GreenER $^{\mathrm{TM}}$ qPCR Supermix for ABI PRISM ${ }^{\circledR}, 300 \mathrm{nM}$ ROX reference dye, $0.2 \mu \mathrm{M}$ forward primer, $0.2 \mu \mathrm{M}$ reverse primer, $15 \mathrm{ng} / \mu \mathrm{L} \mathrm{cDNA}$, and DEPC-treated water in a total volume of $20 \mu \mathrm{L}$. Amplifications involved 40 cycles of PCR reaction and the conditions were: 1 cycle at $95^{\circ} \mathrm{C}$ for $10 \mathrm{~min}$; 40 cycles at $95^{\circ} \mathrm{C}$ for $30 \mathrm{~s}$ and $60{ }^{\circ} \mathrm{C}$ for $1 \mathrm{~min}$; and finally a dissociation stage at $95^{\circ} \mathrm{C}$ for $15 \mathrm{~s}, 60{ }^{\circ} \mathrm{C}$ for $1 \mathrm{~min}$, and $95^{\circ} \mathrm{C}$ for $15 \mathrm{~s}$. The dissociation curve was observed to detect possible primer dimers. Samples were run in triplicate, and a negative control of the Master Mix with primers was performed in all runs.

The Normfinder software (Andersen et al., 2004) was used to identify stable reference genes, and six candidate reference genes including $18 \mathrm{~S}$ ribosomal RNA (18S $r R N A$ ), elongation factor- $1 \alpha$ $(E F 1 a)$, translation elongation factor 2 (TEF2), $\alpha$-tubulin (TUA), ubiquitin extension protein (UBQ5), and polyubiquitin (UBQ10) were optimized for their stability. For validation of the PCR efficiency, the dilution series of different input amounts for cDNA were performed equivalent to $60,30,15$, and $7.5 \mathrm{ng} / \mu \mathrm{L}$ of total RNA. Efficiency analysis was performed using the REST 2009 software V.2.0.13 (Qiagen) (Pfaffl et al., 2002). The crossing point $(C q)$ cycles versus the cDNA concentration input were plotted to calculate the slope (mean $\pm \mathrm{SD} ; n=3$ ). The corresponding realtime efficiencies $(E)$ as shown in Table I were calculated according to the equation $E=10^{(-1 / \text { slope })}-1$.

The relative expression levels were also calculated by the REST 2009 software V.2.0.13. When estimating a sample's expression ratio, an intermediate concentration value was calculated using the following equation:

$$
\begin{aligned}
& \text { concentration }= \\
& \text { efficiency average } C q \text { (control) - average } C q \text { (sample) }
\end{aligned}
$$

To calculate the relative expression, the geometric means of all reference gene concentrations were used according to 


$$
\begin{aligned}
& \text { relative expression }= \\
& \text { concentration of gene of interest } \\
& \text { geometric mean of the concentration } \\
& \text { of all reference genes }
\end{aligned}
$$

The bootstrapping technique was used to provide $95 \%$ confidence intervals for the expression ratios. The relative expression data are presented using Whisker-box plots.

\section{Statistical analysis}

Values are expressed as means \pm standard deviation (SD). Data were analysed by the paired $t$ test using Microsoft Excel. The levels of $99 \%$ and $95 \%$ statistical significance were taken at $P<0.01$ and $P<0.05$, respectively.

\section{Results}

\section{Metabolite profile of M. speciosa}

${ }^{1} \mathrm{H}$ NMR spectroscopy was used as a tool for viewing the profile of abundant metabolites of M. speciosa leaves, in which mitragynine accumulates (Fig. 1). The metabolites were identified based on a database comparing the unique signals of each compound (Kim et al., 2010). A total of 15 metabolites were identified including amino acids, sugars, organic acids, phenolic acids, flavonoids, triterpenes, and iridoids (Table II). In the aliphatic region, almost all the proton signals $\left(\delta_{\mathrm{H}} 0.5-3.0 \mathrm{ppm}\right)$ represented amino ac- ids such as alanine, arginine, leucine, isoleucine, and valine. The exception were the unique four singlet signals between $\delta_{\mathrm{H}} 0.8-1.0 \mathrm{ppm}$ that were assigned to ursolic acid. The proton signals in the sugar region around $\delta_{\mathrm{H}} 3.0-5.5 \mathrm{ppm}$ overlapped. Nevertheless, glucose, sucrose, and xylose were identified among the signals in this region. In the region around $\delta_{\mathrm{H}} 5.5-9.0 \mathrm{ppm}$, protons were detected and identified as being from formic acid, gallic acid, caffeic acid, and epigallocatechin. These signals were present in relatively high intensity. A triplet signal at $\delta_{\mathrm{H}} 9.66 \mathrm{ppm}(\mathrm{H}$-aldehyde) and a doublet signal at $\delta_{\mathrm{H}} 7.48 \mathrm{ppm}(\mathrm{H}-9$ proton in the pyran ring) were interpreted to represent secologanin. From the ${ }^{1} \mathrm{H}$ NMR spectrum, no signals for tryptophan, tryptamine, and mitragynine were detected. The limit of detection and specificity caused some difficulty in identifying all metabolites. The HPLC metabolite analysis thus was performed to detect metabolites involved in mitragynine biosynthesis. Methanol extracts of leaves, roots, and stems of $M$. speciosa cultures were prepared for HPLC analysis.

The optimal conditions for the simultaneous quantitative determination of secologanin, tryptophan, tryptamine, and mitragynine using a gradient reverse phase HPLC system were investigated for the first time in this plant system. All compounds were eluted within 10 min with a satisfactory resolution. The retention times for tryptophan, tryptamine, secologanin, and mitragynine

Table II. ${ }^{1} \mathrm{H}$ NMR chemical shifts and coupling constants of metabolites found in $M$. speciosa leaves, identified by references and using 1D and 2D NMR spectra ${ }^{a}$.

\begin{tabular}{ll}
\hline Metabolite & Selected characteristic NMR signals \\
\hline Alanine & $1.58(\mathrm{~d}, J=7.2 \mathrm{~Hz})$ \\
Arginine & $1.75(\mathrm{~m}), 3.75(\mathrm{t}, J=5.5 \mathrm{~Hz})$ \\
Leucine and isoleucine & $0.96(\mathrm{~d}, J=7.5 \mathrm{~Hz}), 0.98(\mathrm{~d}, J=7.5 \mathrm{~Hz})$ \\
Valine & $1.00(\mathrm{~d}, J=7.0 \mathrm{~Hz}), 1.06(\mathrm{~d}, J=7.0 \mathrm{~Hz})$ \\
$\alpha$-Glucose & $5.18(\mathrm{~d}, J=3.73 \mathrm{~Hz})$ \\
$\beta$-Glucose & $4.57(\mathrm{~d}, J=7.9 \mathrm{~Hz})$ \\
Sucrose & $5.40(\mathrm{~d}, J=3.82 \mathrm{~Hz}), 4.17(\mathrm{~d}, J=8.5 \mathrm{~Hz})$ \\
Xylose & $3.20(\mathrm{~s}), 3.90(\mathrm{~m}), 5.18(\mathrm{~d}, J=3.6 \mathrm{~Hz})$ \\
Formic acid & $8.45(\mathrm{~s})$ \\
Gallic acid & $7.01(\mathrm{~s})$ \\
Caffeic acid & $6.35(\mathrm{~d}, J=15.9 \mathrm{~Hz}), 6.89(\mathrm{~d}, J=8.2 \mathrm{~Hz}), 7.15(\mathrm{~d}, J=1.9 \mathrm{~Hz}), 7.61(\mathrm{~d}, J=15.9 \mathrm{~Hz})$ \\
Ursolic acid & $0.80,0.86,0.90,1.00(\mathrm{~s})$ \\
Epigallocatechin & $4.26(\mathrm{brs}), 2.89(\mathrm{dd}, J=17.0,4.5 \mathrm{~Hz}), 2.73(\mathrm{dd}, J=17.0,2.5 \mathrm{~Hz})$, \\
& $6.02(\mathrm{~d}, J=2.0 \mathrm{~Hz}), 6.05(\mathrm{~d}, J=2.0 \mathrm{~Hz}), 6.59(\mathrm{~s})$ \\
Secologanin & $9.66(\mathrm{t}, J=1.4 \mathrm{~Hz})$ \\
\hline
\end{tabular}

a The NMR spectra were processed and compared to the standards in the database of the Natural Product Laboratory, Leiden, The Netherlands. 
T. Charoonratana et al. · Mitragynine Biosynthesis in Mitragyna speciosa

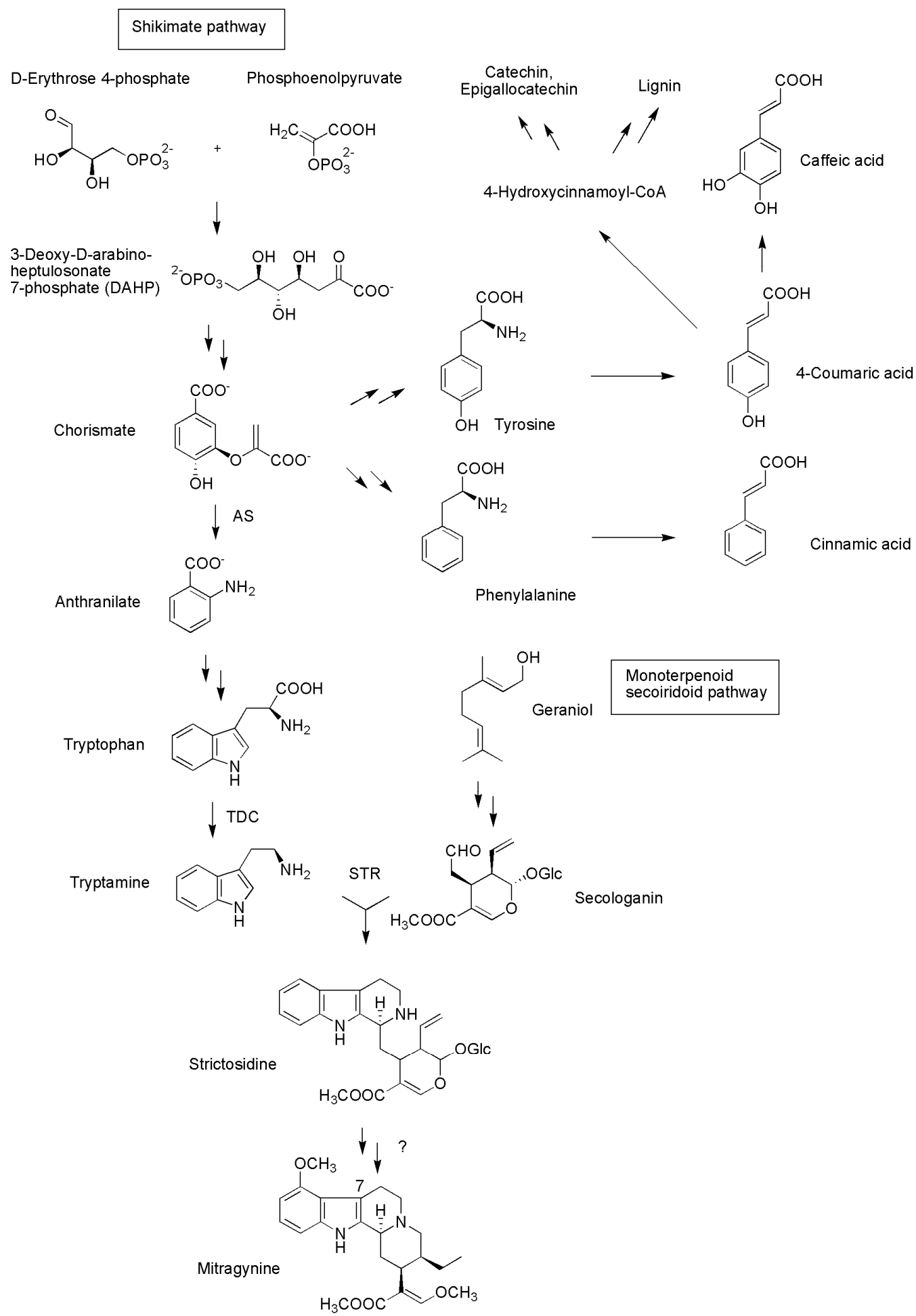

Fig. 1. Proposed mitragynine biosynthetic pathway and of its related metabolites. AS, anthranilate synthase; TDC, tryptophan decarboxylase; STR, strictosidine synthase. 


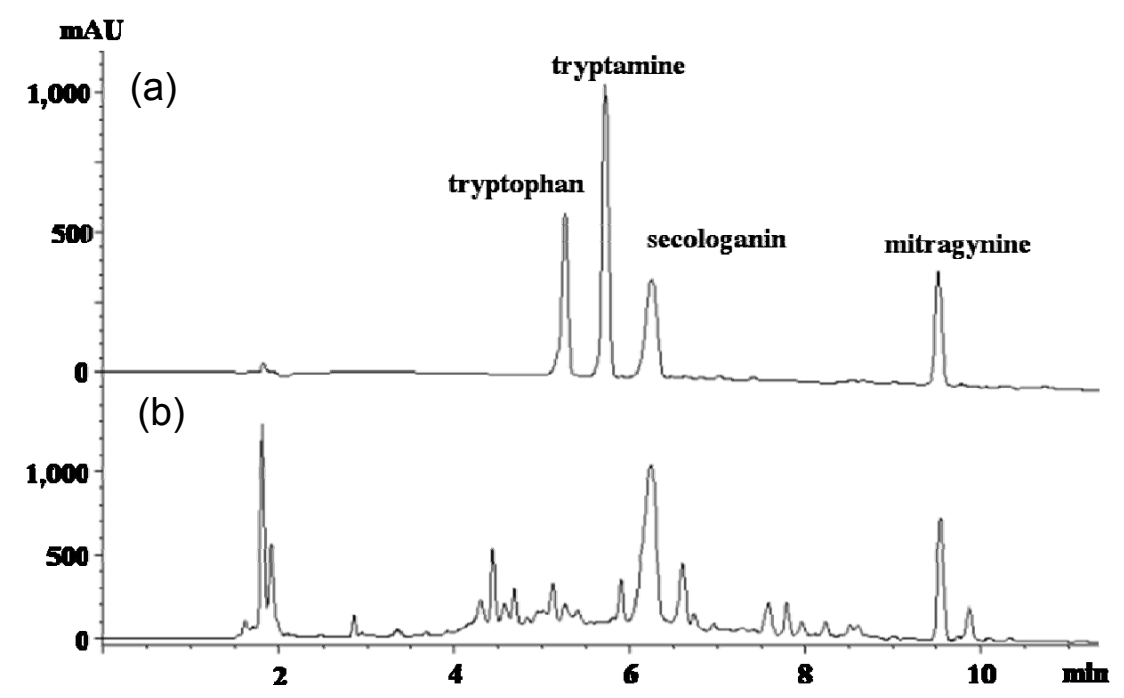

Fig. 2. HPLC chromatograms of (a) authentic compounds and (b) the methanol extract from M. speciosa leaves.

were $5.2 \mathrm{~min}, 5.7 \mathrm{~min}, 6.2 \mathrm{~min}$, and $9.5 \mathrm{~min}$, respectively (Fig. 2). Linearity was examined using authentic standards over five calibration points with six measurements for each calibration point. Secologanin, tryptophan, tryptamine, and mitragynine exhibited good linearity over the evaluated ranges with correlation coefficients of 0.9997 , $0.9999,0.9998$, and 0.9999 , respectively. The precision of the method was assessed by determining the relative standard deviation (\% R.S.D.) of intra- and interday analysis. The method was shown to be reproducible and reliable as both the intraday and interday precisions were lower than $5 \%$. The accuracy of the method was evaluated by analysing the $M$. speciosa leaf extract spiked with known concentrations of the standards. Prior to spiking, the background levels of metabolites were determined so as to calculate actual recoveries. Mean recoveries were in the range of $95-100 \%$ for all compounds. The established HPLC method was found to be very sensitive for tryptophan and tryptamine with a limit of detection (LOD) and limit of quantification (LOQ) of 0.6 and $2.4 \mu \mathrm{g} / \mathrm{mL}$, respectively. The LOD and LOQ for secologanin and mitragynine were 0.2 and $1.2 \mu \mathrm{g} / \mathrm{mL}$, respectively.

The established HPLC procedure was used to determine the content of tryptophan, tryptamine, secologanin, and mitragynine in leaves, roots, and stems of $M$. speciosa. As can be seen from Table III, tryptophan and tryptamine were not detectable in any part of $M$. speciosa. In contrast, secologanin accumulated in considerable amounts in the leaves, followed by smaller amounts in the roots and stems. On the other hand, mitragynine accumulated only in the aerial parts, mainly in the leaves, and was not detected in the roots.

Table III. Metabolite contents in 2-month-old M. speciosa determined by HPLC.

\begin{tabular}{lccc}
\hline Metabolite & \multicolumn{3}{c}{ Content $(\mathrm{mg} / \mathrm{g} \mathrm{DW} \pm \mathrm{SD})^{\mathrm{a}}$} \\
\cline { 2 - 4 } & Leaves & Roots & Stems \\
\hline Tryptophan & n.d. & n.d. & n.d. \\
Tryptamine & n.d. & n.d. & n.d. \\
Secologanin & $9.36 \pm 0.23$ & $4.11 \pm 0.29$ & $4.11 \pm 0.24$ \\
Mitragynine & $3.96 \pm 0.21$ & n.d. & $0.78 \pm 0.07$ \\
\hline
\end{tabular}

aean values \pm standard deviation $(n=3)$.

b n.d., not detected. 
Table IV. Metabolite contents in 2-month-old M. speciosa, supplemented with precursors.

\begin{tabular}{|c|c|c|c|c|c|}
\hline \multirow[t]{3}{*}{ Metabolite } & \multicolumn{5}{|c|}{ Content $(\mathrm{mg} / \mathrm{g} \mathrm{DW} \pm \mathrm{SD})^{\mathrm{a}}$} \\
\hline & \multirow[t]{2}{*}{ Control } & \multicolumn{4}{|c|}{ Fed precursor ${ }^{\mathrm{b}}$} \\
\hline & & Tryptamine & Tryptophan & Tyrosine & Phenylalanine \\
\hline Tryptophan & n.d. ${ }^{\mathrm{c}}$ & n.d. & $0.15 \pm 0.03^{\mathrm{d}}$ & $0.10 \pm 0.01^{\mathrm{d}}$ & $0.09 \pm 0.01^{\mathrm{d}}$ \\
\hline Tryptamine & n.d. & n.d. & n.d. & n.d. & n.d. \\
\hline Secologanin & $9.59 \pm 0.12$ & $9.31 \pm 0.20$ & $9.74 \pm 0.09$ & $9.54 \pm 0.10$ & $9.33 \pm 0.15$ \\
\hline Mitragynine & $3.22 \pm 0.18$ & $6.38 \pm 0.23^{\mathrm{d}}$ & $4.66 \pm 0.15^{\mathrm{d}}$ & $4.20 \pm 0.18^{\mathrm{d}}$ & $4.47 \pm 0.20^{\mathrm{d}}$ \\
\hline
\end{tabular}

a Mean values \pm standard deviation $(n=3)$.

b Each precursor was fed at the concentration of $1 \mathrm{mg} / \mathrm{mL}$.

c n.d., not detected.

d Significance at $P<0.05$ compared with the control group.

\section{Effect of precursor feeding on} mitragynine production

Data from the ${ }^{1} \mathrm{H}$ NMR-based and HPLC-based metabolite analyses, respectively, indicated that there was an excess of the amount of secologanin and no detectable tryptamine in the $M$. speciosa plant model. To assess the limitation on mitragynine biosynthesis, tryptophan and tryptamine were selected, whereas tyrosine and phenylalanine were chosen since they act as precursors for caffeic acid and epigallocatechin (Fig. 1).

After feeding of the amino acids for one month, the newly formed shoots were dissected and prepared for HPLC metabolite analysis. As shown in Table IV, tryptamine was utilized and significantly increased the amount of mitragynine that accumulated by about 2-fold. In addition, tryptophan was also utilized, but to a lesser extent, and the mitragynine contents were enhanced by about 1.4-fold $(P<0.05)$. It can be noted that secologanin was found in comparatively large amounts in all parts.

An additional precursor feeding experiment was performed in $M$. speciosa shoot cultures. Feeding of tryptamine caused an increment of mitragynine production, that was observed after 21 days $(P=0.08)$ (Fig. 3a). In contrast, addition of loganin had no effect on mitragynine production. However, feeding the combination of tryptamine and loganin significantly stimulated mitragynine production within 14 days of feeding $(P<0.01)$. It can be noted that the shoot cultures, fed with this combination for 21 days, contained lower amounts of mitragynine than after 14 days of treatment.

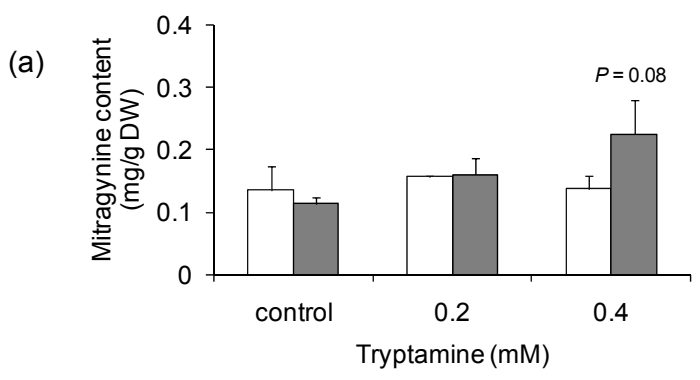

(b)

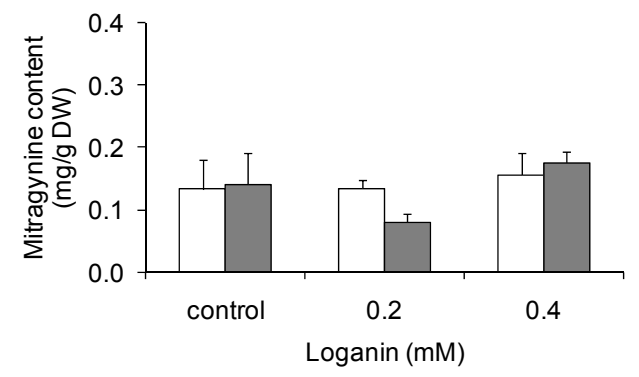

(c)

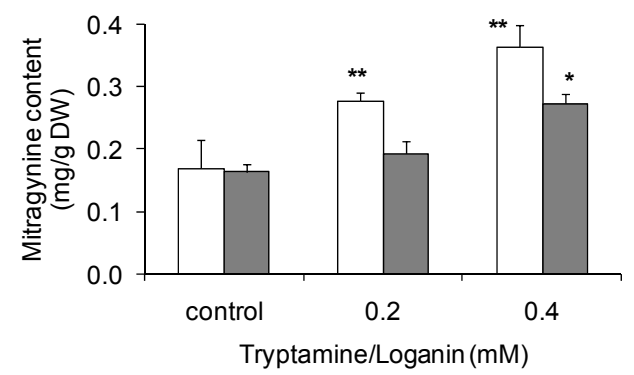

Fig. 3. Mitragynine production in 2-month-old $M$. speciosa shoot cultures fed with (a) tryptamine, (b) loganin, or (c) their combinations for 14 days $(\square)$ and 21 days $(\square)$. $*$ and $* *$ indicate $P<0.05$ and $P<0.01$, respectively. 
Transcription profile of the shikimate pathway genes in M. speciosa

The mRNA levels of shikimate pathway genes, including $A S A 1, A S A 2, T D C$, and $S T R$, of $M$. speciosa were measured under non-induced and MJ-induced (for $12 \mathrm{~h}$ and $24 \mathrm{~h}$ ) conditions, respectively. The transcript levels were determined by RT-qPCR for which a method validation was required. RT-qPCR runs with no-template were included in the control mixture in order to check for the purity of the PCR components and nonspecific amplification. For the design of primers, the Vector NTI $^{\circledR}$ program provided the oligonucleotides with good specificity and efficiency. The sizes of the PCR products were confirmed by agarose gel electrophoresis. Only one derivative was observed in each PCR reaction, and this had a unique melting temperature profile. Thus formation of primer dimers did not occur.

The PCR efficiency was determined using an external standard curve with a serial dilution series of a cDNA template in separate wells. The efficiency values for each well were calculated by the REST 2009 software V.2.0.13, as shown in Table I. In order to find an appropriate normalized gene for the RT-qPCR analysis, the expression stability of the normalized gene was analysed using Normfinder algorithms (Andersen et al., 2004). This method identified the optimum reference gene among groups of candidate genes by taking the data from the estimate of their intra- and intergroup variations. An internal control gene was needed for the normalization strategy for the RT-qPCR (Huggett et al., 2005). Six internal candidate genes were selected. Based on the Normfinder data, a combination of $18 \operatorname{SrRNA}$ and $U B Q 10$ was selected as the reference genes with stability values of 0.586 , which was considered to be medium reference target stability.

According to the validated RT-qPCR method, transcripts of all genes were detected in all samples. The $A S A 2, T D C$, and $S T R$ were expressed in almost equal abundances among stems and leaves, but the expression of $A S A 1$ in the stems was higher than that in the leaves (Fig. 4a). After application of MJ $(10 \mu \mathrm{M})$ to the plant, levels of the transcripts of all genes increased. The increment of the expression appeared to be most prevalent after $12 \mathrm{~h}$ of treatment in the stems and $24 \mathrm{~h}$
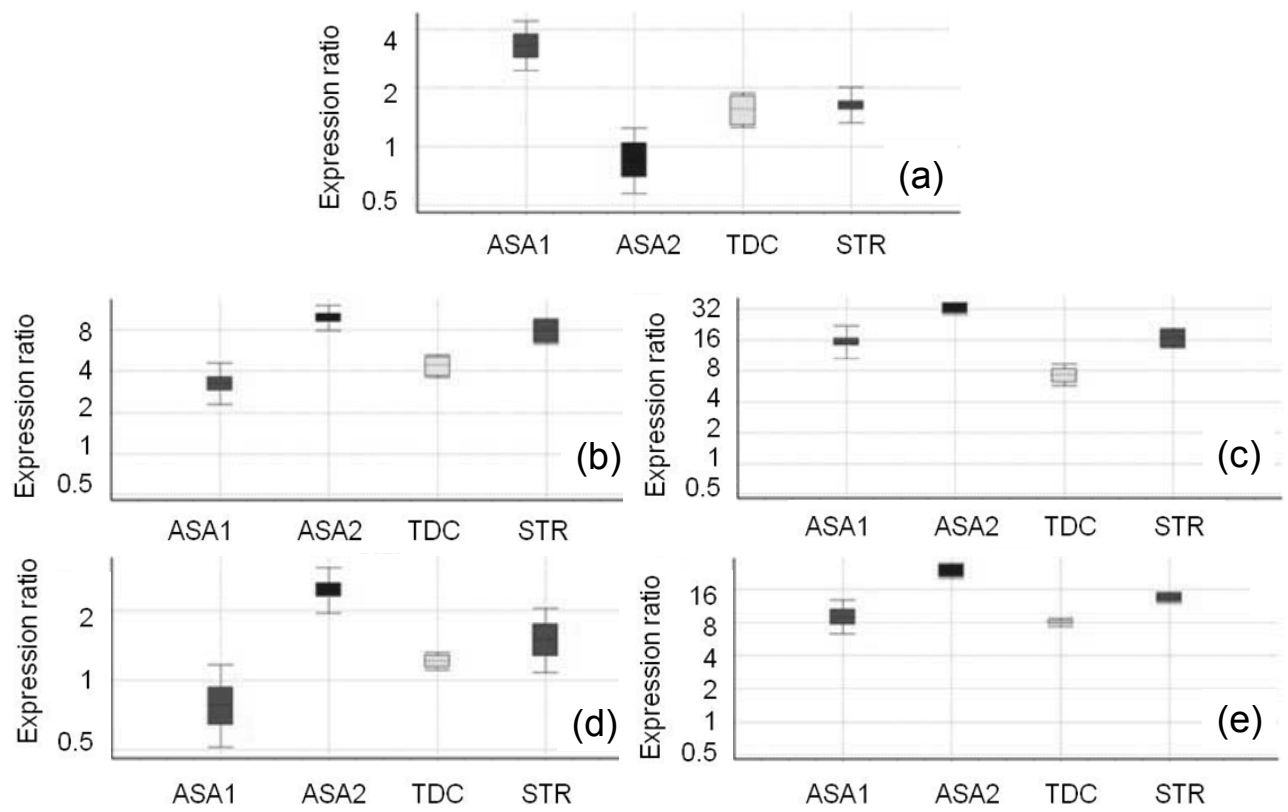

Fig. 4. Whisker-box plot of the relative expressions calculated by the REST 2009 Software of the $A S A 1, A S A 2$, $T D C$, and $S T R$ genes from (a) the stems relative to the leaves of a non-induced group, (b) 12-h MJ-induced stems relative to the non-induced stems, (c) 24-h MJ-induced stems relative to the non-induced stems, (d) 12-h MJinduced leaves relative to the non-induced leaves, (e) 24-h MJ-induced leaves relative to the non-induced leaves. $A S A 1$, anthranilate synthase alpha isoform 1; $A S A 2$, anthranilate synthase alpha isoform 2; TDC, tryptophan decarboxylase; $S T R$, strictosidine synthase. 
in the leaves. After $12 \mathrm{~h}$ of treatment with $\mathrm{MJ}$, transcript levels of $A S A 1$ and $T D C$ were about 4-fold and those of $A S A 2$ and $S T R$ were about 8 -fold higher than in the non-induced stems. All three genes in the stems were significantly expressed (about 8- to 32-fold) after MJ treatment for $24 \mathrm{~h}$ (Figs. 4b, c). As expected, the mitragynine content of the MJ-induced stems was increased significantly $(P<0.05)$ (Table $\mathrm{V})$. This was also apparent in the leaves. MJ induced the expression of all genes by about 8- to 16-fold after $24 \mathrm{~h}$ of treatment (Figs. 4d, e), which was consistent with the enhanced production of mitragynine (Table $\mathrm{V})$. It was noted that the amount of mitragynine in the leaves increased in the first $12 \mathrm{~h}$ and then declined after $24 \mathrm{~h}$ of MJ treatment.

\section{Discussion}

An NMR- and HPLC-based metabolite profiling study was used to identify the biosynthetic profile of mitragynine and related compounds in M. speciosa. The most remarkable outcome, confirmed by both techniques, was the undetectable amount of tryptamine in contrast to the excess amounts of secologanin found in all tissues of M. speciosa. In addition, gallic acid, caffeic acid, epigallocatechin, and ursolic acid were present in relatively high amounts in $M$. speciosa leaves. Caffeic acid is known to be involved in the lignin biosynthesis, whereas gallic acid is a constituent of tannins (Dewick, 2002). Since the biosyntheses of caffeic acid and epigallocatechin utilize chorismate from the shikimate pathway, this means that the flux of primary metabolites to a great

Table V. Mitragynine contents in stems and leaves of control and MJ-induced plants.

\begin{tabular}{ll}
\hline Sample & $\begin{array}{l}\text { Mitragynine } \\
(\mathrm{mg} / \mathrm{g} \mathrm{DW} \pm \mathrm{SD})^{\mathrm{a}}\end{array}$ \\
\hline Stems & \\
Control & $1.22 \pm 0.05$ \\
$12 \mathrm{~h} \mathrm{MJ}$ & $1.46 \pm 0.03^{\mathrm{b}}$ \\
$24 \mathrm{~h} \mathrm{MJ}$ & $1.59 \pm 0.04^{\mathrm{b}}$ \\
\hline Leaves & \\
Control & $4.01 \pm 0.10$ \\
$12 \mathrm{~h} \mathrm{MJ}$ & $4.48 \pm 0.13^{\mathrm{b}}$ \\
$24 \mathrm{~h} \mathrm{MJ}$ & $3.90 \pm 0.09$ \\
\hline
\end{tabular}

a Mean values \pm standard deviation $(n=3)$.

b Significance at $P<0.05$ compared with the control group. extent goes through tyrosine and phenylalanine (Fig. 1). Thus, they compete with the biosynthesis of tryptophan. In order to overcome the possible limitation of substrate availability, the growth medium was supplemented with the mitragynine precursors tryptophan or tryptamine. Tyrosine and phenylalanine were also fed. This revealed that a major limiting factor in mitragynine biosynthesis is the provision of tryptamine, the immediate substrate of strictosidine synthase (STR). The amount of mitragynine was increased significantly in all precursor-supplemented samples, and the most influential precursor was tryptamine. While the actual control point is not known, the feedback inhibition of tyrosine and phenylalanine on chorismate utilization and of tryptophan on anthranilate synthase can be proposed. The existence of ursolic acid indicated that there was a channeling of isoprene units into terpenoid biosynthesis. The accumulation of high amounts of secologanin made the monoterpenoid pathway only a second priority for investigation of the potential of metabolic engineering in $M$. speciosa. This conclusion was confirmed by the results of feeding tryptamine or loganin. Although the metabolite profile of $M$. speciosa shoot culture is different from that of the intact plant, the cultures have the capacity to produce mitragynine (Wungsintaweekul et al., 2012). At $0.2 \mathrm{~mm}$ and $0.4 \mathrm{~mm}$ of tryptamine or loganin, mitragynine production in the shoot culture reacted more sensitively to tryptamine than to loganin, and even more to the combination of both (Fig. 3). The determining factor appeared to be tryptamine.

The role of the shikimate pathway in mitragynine biosynthesis is summarized in Fig. 1. Based on the metabolite profiles and the results of precursor feeding, it is clear that the availability of tryptamine is an important target for further studies on the enhancement of mitragynine yields. Tryptophan decarboxylase (TDC) expression probably plays a crucial role in the rate of the conversion of tryptophan to tryptamine, a substrate of STR. Evidence from the literature indicates that expression of TDC affects MIA accumulation. For instance, induction of ajmalicine formation was observed in low-density Catharanthus roseus cell cultures with concomitant enhancement of TDC activity (Moreno et al., 1993). In Agrobacterium rhizogenes transformed hairy roots from C. roseus, the production of MIAs oc- 
curred only upon induction by MJ, which caused an elevation of $T D C$ transcript levels.

In this study, MJ was also applied to the plants to potentially improve the alkaloid production in $M$. speciosa. It was previously found that MJ induces genes related to MIA biosynthesis. The involvement of an octadecanoid-responsive $\mathrm{Ca}$ tharanthus AP2 domain (ORCA)-like transcription factor in $M$. speciosa can be proposed, since in C. roseus, MJ has been found to induce ORCA transcription factors that bind to the promoters of $T D C$ and other MIA biosynthetic genes, thereby enhancing their transcription and eventually MIA production (Memelink and Gantet, 2007). Recently, CrWRKY1 was identified as another MJ-induced transcription factor in C. roseus hairy roots, and its overexpression resulted in an up to 3-fold increase of the serpentine content in these roots (Suttipanta et al., 2011).

In this study, the mitragynine content increased in stems, which was consistent with the higher expression levels of $A S A$ and $T D C$. The observation that the mitragynine content of leaves declined after 24-h MJ treatment, as well as after feeding tryptamine and loganin for 21 days, suggests that mitragynine may be not the endpoint of al-

Andersen C. L., Jensen J. L., and Orntoft T. F. (2004), Normalization of real-time quantitative reverse transcription-PCR data: a model-based variance estimation approach to identify genes suited for normalization, applied to bladder and colon cancer data sets. Cancer Res. 64, 5245-5250.

Dewick P. M. (2002), Medicinal Natural Product: a Biosynthetic Approach, $2^{\text {nd }}$ ed. John Wiley \& Sons, Chichester, West Sussex, UK.

Goklany S., Loring R. H., Glick J., and Lee-Parsons C. W. T. (2009), Assessing the limitation to terpenoid indole alkaloid biosynthesis in Catharanthus roseus hairy root cultures through gene expression profiling and precursor feeding. Biotechnol. Prog. 25, 1289-1296.

Huggett J., Dheda K., Bustin S., and Zumla A. (2005), Real-time RT-PCR normalization; strategies and considerations. Genes Immunol., 1-6.

Janchawee B., Keawpradub N., Chittrakarn S., Prasettho S., Wararatananurak P., and Sawangjaroen K. (2007), A high-performance liquid chromatographic method for determination of mitragynine in serum and its application to a pharmacokinetic study in rats. Biomed. Chromatogr. 21, 176-183. kaloid biosynthesis in $M$. speciosa. Many MIAs are found at much higher concentrations in leaves as compared to stems of this plant (Takayama, 2004). Mitragynine could be metabolized to other alkaloids or be degraded, but the later steps of mitragynine biosynthesis in $M$. speciosa and its turnover still need to be unraveled.

\section{Acknowledgement}

This research was supported by the Thailand Research Fund (TRF), the Commission of Higher Education (CHE), Prince of Songkla University (PSU), and the Faculty of Pharmaceutical Sciences (Grant No. RMU5380015) and the Annual Government Statement of Expenditure, PSU (Grant No. PHA540039S). Grants from the PSU Graduate School and Songklanakarin Research Abroad to T. C. are also acknowledged. J. W. and T. C. are grateful to the Natural Product Laboratory, Leiden, The Netherlands, for providing facilities for NMR metabolite analysis. Mitragynine was kindly provided by Assistant Prof. Dr. Niwat Keawpradub. We also thank Dr. Kashif Ali for NMR operation and Dr. Brian Hodgson for assistance with the English.
Jumali S. S., Said I. M., Ismail I., and Zainal Z. (2011), Genes induced by high concentration of salicylic acid in Mitragyna speciosa. Aust. J. Crop Sci. 5, 296-303.

Kikura-Hanajiri R., Kawamura M., Maruyama T., Kitajima M., Takayama H., and Goda Y. (2009), Simultaneous analysis of mitragynine, 7-hydroxymitragynine, and other alkaloids in the psychotropic plant "kratom" (Mitragyna speciosa) by LC-ESI-MS. Forensic Toxicol. 27, 67-74.

Kim H. K., Choi Y. H., and Verpoorte R. (2010), NMRbased metabolomic analysis of plants. Nat. Protoc. 5, $536-549$.

Memelink J. and Gantet P. (2007), Transcription factors involved in terpenoid indole alkaloid biosynthesis in Catharanthus roseus. Phytochem. Rev. 6, 353-362.

Moreno P. R. H., Schlatmann J. E., van der Heijden R., Gulik W. M. V., Hoopen H. J. G. T., Verpoorte R., and Heijnen J. J. (1993), Induction of ajmalicine formation and related enzyme activities in Catharanthus roseus cells: effect of inoculum density. Appl. Microbiol. Biotechnol. 39, 42-47.

Nagakura N., Rueffer M., and Zenk M. H. (1979), The biosynthesis of monoterpenoid indole alkaloids from strictosidine. J. Chem. Soc. Perkin Trans. I, 2308-2312. 
Pfaffl M. W., Horgan G. W., and Dempfle L. (2002), Relative expression software tool (REST) for groupwise comparison and statistical analysis of relative expression results in real-time PCR. Nucleic Acids Res. 30, e36.

Ponglux D., Wongseripipatana S., Takayama H., Kikuchi M., Kurihara M., Kitajima M., Aimi N., and Sakai

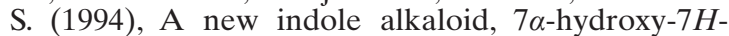
mitragynine, from Mitragyna speciosa in Thailand. Planta Med. 60, 580-581.

Rueffer M., Nagakura N., and Zenk M. H. (1978), Strictosidine, the common precursor for monoterpenoid indole alkaloids with $3 \alpha$ and $2 \beta$ configuration. Tetrahedron Lett. 18, 1593-1596.

Singh G. K. (1932), The pharmacology of mitragynine. J. Pharmacol. 46, 251-271.

Sumner L. W., Mendes P., and Dixon R. A. (2011), Plant metabolomics: large-scale phytochemistry in the functional genomics era. Phytochemistry 62, 817-836.

Suttipanta N., Pattanaik S., Kulshrestha M., Patra B., Singh S. K., and Yuan L. (2011), The transcription factor CrWRKY1 positively regulates the terpenoid indole alkaloid biosynthesis in Catharanthus roseus. Plant Physiol. 157, 2081-2093.

Suwanlert S. (1975), A study of kratom eaters in Thailand. Bull. Narc. 27, 21-27.

Takayama H. (2004), Chemistry and pharmacology of analgesic indole alkaloids from the Rubiaceous plant, Mitragyna speciosa. Chem. Pharm. Bull. 52, 916-928.

Thongpraditchote S., Matsumoto K., Tohda M., Takayama H., Aimi N., Sakai S., and Watanabe H. (1998), Identification of opioid receptor subtypes in antinociceptive actions of supraspinally-administered mitragynine in mice. Life Sci. 62, 1371-1378.

Wungsintaweekul J., Choo-malee J., Charoonratana T., and Keawpradub N. (2012), Methyl jasmonate and yeast extract stimulate mitragynine production in Mitragyna speciosa (Roxb.) Korth. shoot culture. Biotechnol. Lett. 34, 1945-1950. 